Notice: This manuscript has been authored by UT-Battelle, LLC, under Contract No. DEAC0500OR22725 with the U.S. Department of Energy. The United States Government retains and the publisher, by accepting the article for publication, acknowledges that the United States Government retains a non-exclusive, paid-up, irrevocable, world-wide license to publish or reproduce the published form of this manuscript, or allow others to do so, for the United States Government purposes. The Department of Energy will provide public access to these results of federally sponsored research in accordance with the DOE Public Access Plan (http://energy.gov/downloads/doe-public-access-plan). 


\section{Controlling the Actuation Properties of MXene Paper Electrodes upon Cation Intercalation}

Jeremy Come, ${ }^{a}$ Jennifer M. Black, ${ }^{a}$ Maria R. Lukatskaya,${ }^{b}$ Michael Naguib ${ }^{c}$ Majid Beidaghi, ${ }^{b}$ Adam J. Rondinone, ${ }^{a}$ Sergei V. Kalinin, ${ }^{a}$ David J. Wesolowski, ${ }^{d}$ Yury Gogotsi,${ }^{b}$ and Nina Balke ${ }^{a,{ }^{*}}$

${ }^{a}$ Center for Nanophase Materials Sciences, Oak Ridge National Laboratory, P.O. Box 2008, Oak

Ridge, TN, 37831-6496, USA

${ }^{b}$ Department of Materials Science and Engineering \& A. J. Drexel Nanotechnology Institute, Drexel University, Philadelphia, PA, 19104, USA

${ }^{c}$ Materials Science and Technology Division, Oak Ridge National Laboratory, Oak Ridge, TN, 37831, USA

${ }^{d}$ Chemical Sciences Division, Oak Ridge National Laboratory, Oak Ridge, TN, 37831, USA

Jeremy Come - email: comeje@ ornl.gov

Jennifer M. Black - email: $\underline{\text { blackjm@ornl.gov }}$

Maria R. Lukatskaya - email: $\underline{\text { mrl69@ drexel.edu }}$

Michael Naguib - email : naguibma@ornl.gov

Majid Beidaghi - email: mbeidaghi@coe.drexel.edu

Adam J. Rondinone - email : rondinoneaj@ornl.gov

Sergei V. Kalinin - email: $\underline{\text { sergei2@ornl.gov }}$

David J. Wesolowski - email: wesolowskid@ornl.gov

Yury Gogotsi - email: gogotsi@drexel.edu

* Nina Balke (corresponding author) - email: balken@ornl.gov, ph: +1(865)-241-5470 


\begin{abstract}
Atomic force microscopy was used to monitor the macroscopic deformation in a delaminated $\mathrm{Ti}_{3} \mathrm{C}_{2}$ paper electrode in situ, during charge/discharge in a variety of aqueous electrolytes to examine the effect of the cation intercalation on the electrochemical behavior and mechanical response. The results show a strong dependence of the electrode deformation on cation size and charge. The electrode undergoes a large contraction during $\mathrm{Li}^{+}, \mathrm{Na}^{+}$or $\mathrm{Mg}^{2+}$ intercalation, differentiating the $\mathrm{Ti}_{3} \mathrm{C}_{2}$ paper from conventional electrodes where redox intercalation of ions (e.g. $\left.\mathrm{Li}^{+}\right)$into the bulk phase (e.g. graphite, silicon) results in volumetric expansion. This feature may explain the excellent rate performance and cyclability reported for MXenes. We also demonstrated that the variation of the electromechanical contraction can be easily adjusted by electrolyte exchange, and shows interesting characteristics for the design of actuators based on 2D metal carbides.
\end{abstract}

Keywords: MXene; intercalation; electromechanical actuator; atomic force microscopy. 


\section{Introduction}

As the demand for renewable and clean energy sources is ever increasing, interest in electrochemical energy storage devices such as lithium ion batteries and electrochemical capacitors (ECs) has risen in recent years. ECs have received much attention due to their high power densities but they suffer from low energy densities [1-3]. ECs can be categorized into two types: i) electric double layer capacitors (EDLCs) that store charge by electrostatic adsorption of ions on the surface of porous conducting materials like carbon, and ii) pseudo-capacitors that store charge by means of fast surface redox reactions [4]. Recently it was shown that fast intercalation of ions between atomic layers of some crystalline transition metal oxides [5] or layered two-dimensional (2D) materials such as the titanium carbide $\mathrm{Ti}_{3} \mathrm{C}_{2} T_{x}$ (where $T_{x}$ stands for surface terminations such as $\mathrm{OH},=\mathrm{O}$ and F) [6] can result in high volumetric capacitance. The fast intercalation of ions in these materials happens with no diffusion limitations, which is a key requirement for supercapacitor electrodes. This material is a member of the recently discovered family of 2D materials known as MXenes [7-11]. These materials store energy by rapid ion intercalation between the $2 \mathrm{D}$ sheets, as well as $\mathrm{Ti}^{4+} / \mathrm{Ti}^{3+}$ and other surface redox reactions, making them promising ECs electrodes $[12,13]$.

In situ X-ray diffraction and Electrochemical Quartz Crystal Admittance (EQCA) measurements during charge/discharge suggested that cation intercalation between MXene sheets leads to a decrease in the distance between the layers (and corresponding $c$-lattice parameter) [6,14]. Depending on the charge/ionic radius ratio of the cation, the screening of electrostatic repulsive forces between charged groups on the $\mathrm{Ti}_{3} \mathrm{C}_{2} T_{x}$ sheet surfaces may result in different electrode deformation. Such a feature is appealing for electrochemically-controlled actuators, which convert electrical energy into mechanical energy $[\mathbf{1 5 , 1 6 ]}$. Moreover, thanks to its metallic 
electrical conductivity [17], as well as its high predicted elastic constants and bending rigidity [18], $\mathrm{Ti}_{3} \mathrm{C}_{2} T_{x} \mathrm{MXene}$ appears to be a good candidate for electromechanical applications. Yet, no reports on direct measurements of the MXene deformation are available, and the effective macroscopic volumetric changes of the electrode during charge and discharge have not been described so far. Furthermore, understanding the intricate interplay of ion insertion, electrode volume changes, and the resulting capacitance can help to optimize and tailor the electrochemical performance and lifetime of electrode materials.

In this work we investigate the macroscopic deformation of the $\mathrm{Ti}_{3} \mathrm{C}_{2} T_{x}$ "paper" (additives-free assembly of the few/single-layer(s) MXene flakes shown in Fig.1a) during cation insertion (charging). Atomic force microscopy (AFM) has the unique advantage of having a $\mathrm{Z}$ (height) resolution of $<1 \mathrm{~nm}$ and high lateral resolution determined by the AFM tip geometry (ca. $20 \mathrm{~nm}$ ). This technique has been broadly used as an in situ probe to monitor the dimensional changes of battery [19-23], pseudocapacitor [24,25], and EDLC electrodes [26,27]. Another key advantage of these experiments is that they can be performed at various charging and discharging rates. Here, the deformation response of $\mathrm{Ti}_{3} \mathrm{C}_{2} T_{x}$ is measured in different aqueous electrolytes to determine the effect of varying the electrolyte cation size and charge on the electrochemical and mechanical response.

\section{Material and Methods}

\subsection{MXene Synthesis}

Delaminated $\mathrm{Ti}_{3} \mathrm{C}_{2} T_{x}$ electrode synthesis and preparation are described elsewhere [6]. In short, $\mathrm{Ti}_{3} \mathrm{C}_{2} T_{x}$ was synthesized by etching $\mathrm{Al}$ from $\mathrm{Ti}_{3} \mathrm{AlC}_{2}$ using $48 \%$ concentrated hydrofluoric acid at room temperature (RT) for $18 \mathrm{~h}$. After washing to remove the etching products followed by 
drying, $\mathrm{Ti}_{3} \mathrm{C}_{2} T_{x}$ powder was intercalated by dimethylsulfoxide (DMSO). Then, the intercalated MXene powder was sonicated in deionized water and centrifuged. The resulted colloidal solution of delaminated MXene was vacuum-filtered to produce free-standing MXene "paper". The latter, consisting of parallel oriented flakes (Figs.1a and b), was used directly as the working electrode (mass density of $4 \mathrm{mg} \mathrm{cm}^{-2}$ and thickness of $25 \pm 2 \mu \mathrm{m}$ ).

\subsection{Electrochemical Characterization}

Electrochemical experiments were performed using a Bio-Logic SP-200 potentiostat (Bio-Logic, USA). A three-electrode commercial in situ electrochemical AFM cell (Asylum Research, Oxford Instrument Company, USA) was used in this study and is shown in Figs.1c. A $14 \mathrm{~mm}^{2} \mathrm{Ti}_{3} \mathrm{C}_{2} T_{x}$ paper used as the working electrode was attached to a glassy carbon current collector using colloidal graphite and clamped with a PolyEtherEtherKetone (PEEK) cap to ensure a good electrical contact. A $12 \mathrm{~cm}^{2}$ activated carbon ring was used as the counter electrode (1300 $\mathrm{m}^{2} \mathrm{~g}^{-1}$, Y-Carbon, USA). The electrolytes used in this study were $1 \mathrm{M} \mathrm{Li}_{2} \mathrm{SO}_{4}$, $1 \mathrm{M} \mathrm{Na}_{2} \mathrm{SO}_{4}, 0.5 \mathrm{M} \mathrm{K}_{2} \mathrm{SO}_{4}$, and $1 \mathrm{M} \mathrm{MgSO}_{4}(\geq 99.0 \%$, all purchased from Sigma Aldrich, USA).

Cyclic voltammetry (CV) curves were recorded at sweep rates between 1 and $20 \mathrm{mV} \mathrm{s}^{-1}$. Prior to AFM measurements, each working electrode was precycled at $10 \mathrm{mV} \mathrm{s}^{-1}$ for $24 \mathrm{~h}$ to reach a steady state. All potentials are vs $\mathrm{Ag} / \mathrm{AgCl}$ reference electrode. The potential range for electrochemical characterization was chosen to avoid unwanted reactions such as MXene oxidation at high potentials or hydrogen evolution at low potentials.

\subsection{Atomic Force Microscopy}

The relative electrode deformation was monitored using an MFP-3D AFM (Asylum Research, Oxford Instrument Company, USA). Measurements were conducted by keeping the AFM tip in contact with the working electrode with a constant contact force while performing 
$\mathrm{CV}$ at various sweep rates (see Fig. 1d). Pt coated Nanosensors ${ }^{\mathrm{TM}}$ (PPP-EFM-50, $\mathrm{k}=0.5-9.5 \mathrm{~N} \mathrm{~m}^{-1}$ ) AFM tips were used.

\subsection{X-ray diffraction}

X-ray diffraction (XRD) experiments on the $\mathrm{Ti}_{3} \mathrm{C}_{2} T_{x}$ paper were performed on a Panalytical X'Pert diffractometer under copper K-alpha radiation. The electrochemical cell was charged and discharged by linear sweep voltammetry at $1 \mathrm{mV} \mathrm{s}^{-1}$. The potential was held during 20 minutes before collecting the XRD patterns.

\section{Results and Discussion}

Figures $2 \mathrm{a}$ and $\mathrm{b}$ show the $\mathrm{CV}$ curves and the corresponding deformation profile demonstrated by the $\mathrm{Ti}_{3} \mathrm{C}_{2} T_{x}$ paper electrode during intercalation of various cations. Interestingly, insertion of $\mathrm{Li}^{+}, \mathrm{Na}^{+}$and $\mathrm{Mg}^{2+}$ ions results in contraction of the electrode, whereas for $\mathrm{K}^{+}$ electrolyte a small expansion is observed. The values of the relative height change scale appropriately with the charge/ionic radius ratio values $\left(1.67 \AA^{-1}, 1.05 \AA^{-1}, 0.75 \AA^{-1}\right.$ and $2.78 \AA^{-1}$ for $\mathrm{Li}^{+}, \mathrm{Na}^{+}, \mathrm{K}^{+}$and $\mathrm{Mg}^{2+}$, respectively) [28]. For monovalent cations, the contraction amplitude decreases for larger ionic radius, since the steric hindrance contribution due to larger size becomes more important. The attractive forces between negatively charged MXene sheets and cations in the interlayer spaces are likely to dominate the deformation, as represented in Fig $2 \mathrm{c}$. In $\mathrm{K}^{+}$case however, the steric occupancy between MXene sheets dominates the charge storage due to the large ionic radius. It is interesting to note that the effective radii of hydrated ions ( $c a$. $3.82 \AA, 3.58 \AA, 3.31 \AA$ and $4.28 \AA$ for $\mathrm{Li}^{+}, \mathrm{Na}^{+}, \mathrm{K}^{+}$and $\mathrm{Mg}^{2+}$, respectively) [29] do not correlate with the deformation profiles: the larger size of the hydrated $\mathrm{Li}^{+}$ion should result in a smaller contraction than hydrated $\mathrm{Na}^{+}$or $\mathrm{K}^{+}$ions. Most likely, the hydration shell is partially removed or 
rearranged when ions are intercalated between the layers $\left[\mathbf{1 4 , 2 9 , 3 0 ]}\right.$. Hence adsorption of $\mathrm{Li}^{+}$ ions, 4-coordinated in water, results in stronger interactions with the MXene layers than $\mathrm{Mg}^{2+}$ (6-coordinated), leading to a larger contraction.

Interestingly, two different regions are clearly observed in the $\mathrm{CV}$ curves and height change profile when the MXene paper is cycled in $\mathrm{Li}_{2} \mathrm{SO}_{4}$. At potentials higher than $-0.25 \mathrm{~V}, \mathrm{Li}^{+}$ intercalation capacitance is higher than $300 \mathrm{~F} \mathrm{~cm}^{-3}$ and it drops to around $200 \mathrm{~F} \mathrm{~cm}^{-3}$ when E $<-0.25 \mathrm{~V}$. At the same time, the electrode contraction is small at first and increases strongly when the capacitance changes during the charging process. During discharge, the contraction rate is small at first and increases strongly again when the capacitance changes, resulting in a hysteresis between charge and discharge (Fig. 2b).

The decrease in the $\mathrm{Li}^{+}$intercalation capacitance along with the change of contraction rate at potentials lower than $-0.25 \mathrm{~V}$ remains unclear at this point, and contrasts with the increase in the capacitance in the same potential region for $\mathrm{Mg}^{2+}$ intercalation. Nevertheless, this strongly points to a 2 steps storage process, either due to adsorption in deep and shallow sites, or to reorganization/ordering of the layered structure. In turn, the hysteresis could be associated with the large positive overpotential required to extract strongly bonded cations that may be exchanging with protons on MXene surface, or to a partial intercalation of anions at potentials higher than $0 \mathrm{~V}$. In order to shed light on this observation, in situ XRD measurements were performed. The XRD pattern of the dry $\mathrm{Ti}_{3} \mathrm{C}_{2} T_{x}$ paper (Fig. 3a) shows the flakes ordering in the $c$ direction. The (0002) peak is located at $8.83^{\circ}$, corresponding to a $d$-spacing of $10.04 \AA$. Figure 3 b shows that the (0002) peak shifted to lower angles during $\mathrm{Li}^{+}$intercalation, and shifted back to higher angles as $\mathrm{Li}^{+}$ions are extracted. As expected, the corresponding $d$-spacing values in Figure $3 \mathrm{~d}$ show the shrinkage of the MXene layers upon $\mathrm{Li}^{+}$intercalation, from $12.84 \AA$ to 
$12.56 \AA$, which is equivalent to $\sim 2.1 \%$. Since the decrease in the $d$-spacing is lower than the macroscopic contraction recorded previously, additional electrostatic forces must play a role in the interactions between MXene sheets. The volume change profile is consistent with the dilatometry experiments, with the same hysteresis shape between charge and discharge. Upon cation intercalation, the diffraction peak becomes sharper and more intense, highlighting that the carbide layers become more ordered as the $\mathrm{Li}^{+}$content increases. As a comparison similar experiments were performed in $\mathrm{K}_{2} \mathrm{SO}_{4}$ electrolyte (Figure $3 \mathrm{c}$ ). Here again the $d$-spacing evolution matches the macroscopic deformation profile, since only $0.3 \%$ volume change was measured. In this case as well the peak shows higher intensity at larger $\mathrm{K}^{+}$content. Surprisingly, intercalation of large $\mathrm{K}^{+}$ion led to smaller $d$-spacing than $\mathrm{Li}^{+}$. Although the reason for this is unclear at this point, the hydration shell structure, the possibility of anion co-intercalation and/or changes in the MXene oxidation state can contribute to variations in the interlayer distances.

The unusual electrode contraction differs from the well-established swelling of other 2D materials such as carbon [31-33] or reduced graphene oxide [34] when they are intercalated with molecules and/or ions. Moreover, the amplitude of the shrinkage can be higher than $10 \%$ in the case of intercalation of $\mathrm{Li}^{+}$. To get more insight in the two-step process observed for $\mathrm{Li}^{+}$ intercalation, Figures $4 \mathrm{a}$ and $\mathrm{b}$ show the $\mathrm{CV}$ curves and corresponding height change profiles plotted versus normalized time for scan rates from 1 to $20 \mathrm{mV} \mathrm{s}^{-1}$. As the capacitance decreases with increasing sweep rate, the electrode undergoes important deformation changes. During intercalation, the electrode first expands (Fig. 4b), and the amplitude of the expansion increases with the sweep rate. The change in the electrode deformation may be the result of complex interactions between $\mathrm{Ti}_{3} \mathrm{C}_{2} T_{x}$ sheets and adsorbed metal ions. At high rates, adsorption of the positively charged cations at the $\mathrm{Ti}_{3} \mathrm{C}_{2} T_{x}$ surface may not be fast enough to shield the 
electrostatic repulsions between negatively charged MXene sheets. The subsequent contraction is measured once a sufficient number of $\mathrm{Li}^{+}$ions are intercalated. This is consistent with the slight expansion observed at high rate during the $\mathrm{Li}^{+}$extraction process, caused by repulsive interactions between the residual cations and the positively charged layers. Such mechanism can contribute to the important capacitance loss at high sweep rates. Overcrowding by the presence of water molecules and possibly anions between the layers may also contribute to the expansion at high rates. Such species might not have enough time to make room for cation adsorption, hence leading to steric hindrance between MXene layers.

The investigation of electrode deformation reveals interesting insights into the intercalation process for cations of different sizes and charges and their interaction with the MXene sheets. Due to the high reproducibility of the observed behavior, it can also be used to tailor the electrode deformation for specific applications. For example, the insertion of $\mathrm{K}^{+}$ions reveals an almost zero electrode deformation which will guarantee low mechanical stresses during charge and discharge, important for a long cyclic lifetime. Cyclability is an important issue for energy storage materials, since the volume changes associated with the electrochemical reaction are often responsible for the capacity fading, as for example $\mathrm{Li}^{+}$intercalation in metal oxides $[35,36]$ or Li alloying reaction with metal anodes $[19,37]$. One of the principal benefits of low volume changes or even contraction materials is that large number of charge/discharge cycles can be achieved along with minimal capacitance loss. Interestingly, electrodes with different interlayer distances can easily be obtained, with the advantage of keeping the electronic conductivity metallic, as well as increasing both the tensile strength and the capacitance values. To date, such MXene-based composites have been fabricated with carbon nanotubes (CNTs), reduced graphene oxide (rGO), onion-like carbon (OLCs) [38], polyvinyl alcohol (PVA) or 
polydiallyldimethylammonium chloride (PDDA) [39]. In each case the interlayer spacing strongly depends on the nature and quantity of the hosted intercalant, and different deformation behaviors are expected for each material. With such a variety of composites in hands, designing a proper electrode/electrolyte couple becomes more accessible to further increase the cycling stability.

On the other side, certain electrochemical capacitors are considered attractive for mechanical actuation applications [40]. Depending on the actuation requirements, the need to change the actuation properties during utilization may arise. To investigate the tunability of the electrochemical capacitor deformation for actuator applications, the same paper electrode was cycled after the electrolyte was switched in the electrochemical cell in the following order: $1 \mathrm{M}$ $\mathrm{MgSO}_{4}, 1 \mathrm{M} \mathrm{Li}_{2} \mathrm{SO}_{4}, 0.5 \mathrm{M} \mathrm{K}_{2} \mathrm{SO}_{4}$ and $1 \mathrm{M} \mathrm{MgSO}_{4}$. Figures $5 \mathrm{a}$ and $\mathrm{b}$ show the $\mathrm{CV}$ curves and corresponding electrode deformation profiles recorded after each electrolyte switch, respectively. The typical electrochemical signature for $\mathrm{Li}^{+}$intercalation along with its height change profile confirms that $\mathrm{Li}^{+}$ions replace $\mathrm{Mg}^{2+}$ ions in the $2 \mathrm{D}$ spaces between the MXene sheets. When $\mathrm{K}^{+}$ ions are subsequently intercalated, the typical CV curve is observed, with very small electrode deformation (compare with Fig. 2b). Finally, when $\mathrm{Mg}^{2+}$ ions are intercalated again, the CV curves show a less resistive behavior and the relative electrode height change, although of lower amplitude, shows the same profile. The faster $\mathrm{Mg}^{2+}$ transport between MXene sheets may come from several effects including i) different adsorption energies due to residual $\mathrm{Li}^{+}$and/or $\mathrm{K}^{+}$ cations, or ii) larger interlayer spaces created by large intercalated $\mathrm{K}^{+}$ions, making more room for $\mathrm{Mg}^{2+}$ transport in the $2 \mathrm{D}$ channels. This demonstrates that the actuation properties can be recovered when switching the electrolyte, enabling to tune the mechanical behavior of the MXene. It also shows a pathway to optimize the intercalation of multivalent ions of bigger size 
for increased charge storage. Even though mechanical deformation tests were not performed in a traditional setup, the observed behavior of MXene paper upon adsorption of cations shows its unique actuation properties and promises for applications such as controllable artificial muscles. Strong free-standing MXene electrodes with gel electrolyte were recently reported and can be used as actuators, especially when combined with easily expanding rGO paper electrodes (Figs.6a and b) [39]. Moreover, controlling the actuation behavior of a device by simple switching of the electrolyte can be a great asset for electromechanical applications (Fig.6c).

\section{Conclusions}

In situ monitoring of the $\mathrm{Ti}_{3} \mathrm{C}_{2} T_{x}$ paper electrode deformation during electrochemical intercalation of various cations using AFM showed that charging of MXene electrode in $\mathrm{Li}^{+}, \mathrm{Na}^{+}$ and $\mathrm{Mg}^{2+}$ electrolytes results in contraction, or light expansion, in the case of $\mathrm{K}^{+}$. This was confirmed by in situ XRD measurements. Although further experiments must be performed to fully understand the charge storage mechanism of the different cations, we can conclude that $\mathrm{Ti}_{3} \mathrm{C}_{2} T_{x}$ MXene electrode deformation strongly depends on the charge/ionic radius ratio. The

unusual reversible contraction of the $\mathrm{Ti}_{3} \mathrm{C}_{2} T_{x}$ electrode during $\mathrm{Li}^{+}$and $\mathrm{Mg}^{2+}$ adsorption may enable the MXene paper to sustain a very large number of charging cycles. Finally, delaminated $\mathrm{Ti}_{3} \mathrm{C}_{2} T_{x}$ paper shows promising properties for asymmetric electromechanical actuators, which can be tuned to cater to specific actuation requirements. 


\section{Figure captions}

Figure 1: a) Scanning electron microscopy image and b) tapping mode AFM image of the surface of the delaminated $\mathrm{Ti}_{3} \mathrm{C}_{2} T_{x}$ paper electrode, c) picture of the electrochemical cell used for in situ volume change measurements, and d) cross-section schematic representation of the cell showing how the MXene is electrically connected and how the AFM tip is used to monitor the electrode deformation in the presence of electrolyte.

Figure 2: a) $\mathrm{CV}$ curves at $2 \mathrm{mV} \mathrm{s}^{-1}$ of $\mathrm{Ti}_{3} \mathrm{C}_{2} T_{x}$ paper in various electrolytes, and b) corresponding relative electrode deformation profiles, c) schematic representation of 2 MXene layers attracted to each other when cations are intercalated in-between $(-\mathrm{OH}$ terminations groups are shown as an example).

Figure 3: a) X-ray diffraction patterns of the dry $\mathrm{Ti}_{3} \mathrm{C}_{2} \mathrm{~T}_{\mathrm{x}}$ paper, evolution of the (0002) diffraction peak at different potentials during electrochemical cycling in b) $1 \mathrm{M} \mathrm{Li} \mathrm{SO}_{4}$ and c) $1 \mathrm{M} \mathrm{K}_{2} \mathrm{SO}_{4}$ electrolytes, and d) corresponding $d$-spacing values as a function of the potential.

Figure 4: a) CV curves from 1 to $20 \mathrm{mV} \mathrm{s}^{-1}$ and b) corresponding height change profiles plotted as a function of normalized time in $\mathrm{Li}_{2} \mathrm{SO}_{4}$ electrolyte.

Figure 5: a) $\mathrm{CV}$ curves at $2 \mathrm{mV} \mathrm{s}^{-1}$, b) corresponding relative deformation profiles in different electrolytes for the same $\mathrm{Ti}_{3} \mathrm{C}_{2} T_{x}$ paper electrode.

Figure 6: Potential examples of actuators based on MXene paper electrode, a) electrical switch, b) micro elevator, or c) circuit breaker that shrinks or expands depending on the chosen electrolyte. 


\section{Vitae}

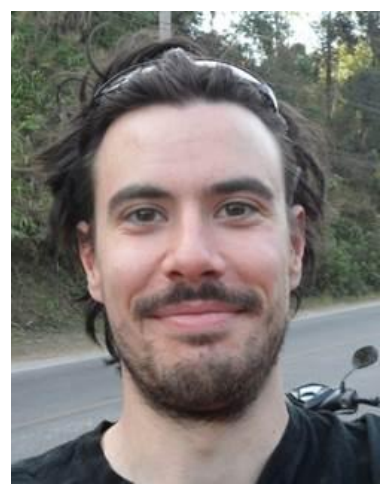

Jeremy Come is a postdoctoral research associate at the Center for Nanophase Materials Sciences (CNMS) at Oak Ridge National Laboratory. He received his Ph.D.in Materials Science from University Paul Sabatier of Toulouse in 2012, and has a European M.Sc. degree in Materials for energy storage and conversion from Amiens, Marseille, Toulouse, Warsaw and Cordoba Universities. His current research interests include the investigation of charge storage mechanisms in capacitive and pseudocapacitive materials using in-situ characterization techniques such as scanning probe microscopy, electronic microscopy or X-ray diffraction.

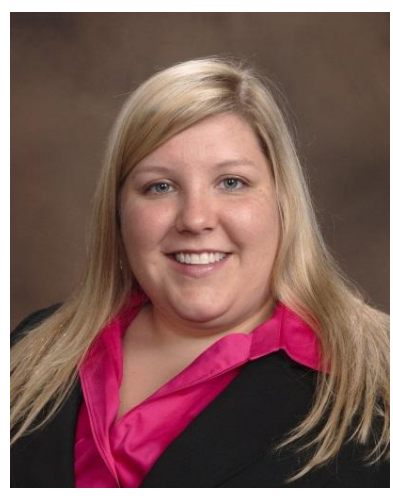

Jennifer Black received her B.Sc. in Chemistry from Cape Breton University in 2006. She did her Ph.D. at Dalhousie University under the supervision of Professor Heather Andreas focusing on the self-discharge mechanisms of electrochemical double-layer capacitors. From 2011-2012 she worked as a postdoctoral researcher at the Ohio State University in the group of Dr. Anne Co researching anode materials for lithium ion batteries and developing metallic foams for catalysis. She joined Oak Ridge National Laboratory as a postdoctoral researcher in 2012 where her current research focuses on the nanoscale characterization of energy storage materials using scanning probe microscopy.

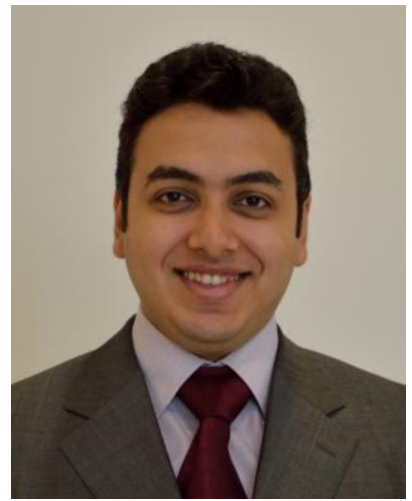

Michael Naguib is a Wigner Fellow at Oak Ridge National Laboratory. He obtained his $\mathrm{PhD}$ in Materials Science and Engineering from Drexel University, Philadelphia, PA, USA. He received his MS and BS degrees in Metallurgical Engineering from the Faculty of Engineering, Cairo University, Egypt. His research focuses on the synthesis and characterization of functional nanomaterials for energy storage. He has published 28 papers in international journals and is a co-inventor on the MXene patent. He has received many international awards, such as MRS Gold Graduate Student Award, Graduate Excellence in Materials Science (GEMS) Award, and Ross Coffin Purdy Award. 


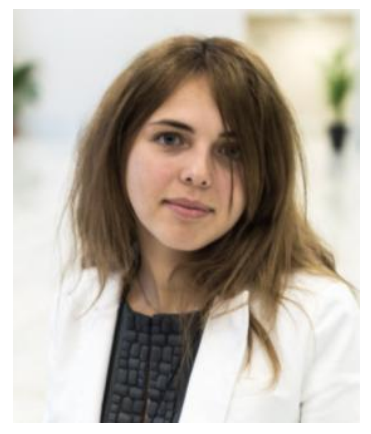

Maria Lukatskaya is a Ph.D. candidate and research assistant in the Department of Materials Science and Engineering at Drexel University. She received her B.Sc. in Materials Science and M.Sc. in Chemistry from Lomonosov Moscow State University, Russia. Her research interests lie in the new nanomaterials and electrochemical system design for energy storage applications. She has published 18 papers in international journals, in addition to presenting oral presentations and posters in many international conferences.

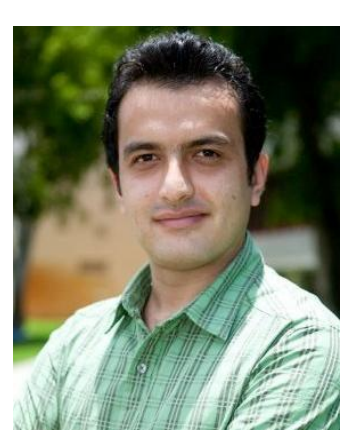

Majid Beidaghi is a Research Associate at A. J. Drexel nanotechnology Institute, Department of Material Science and Engineering at Drexel University. He received his $\mathrm{PhD}$ in Materials Science and Engineering from Florida International University in 2012. His research interests are in the field of electrochemical energy storage with the main focus on the synthesis of multifunctional materials and design and fabrication of electrochemical energy storage devices such as electrochemical capacitors and batteries.

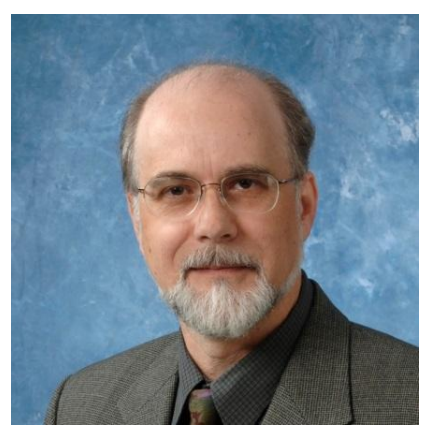

David J. Wesolowski is a Distinguished R\&D Staff Member in the Chemical Sciences Division at Oak Ridge National Laboratory. He is the Leader of the Geochemistry and Interfacial Sciences Group, and the Director of the Fluid Interface Reactions, Structures and Transport (FIRST) Energy Frontier Research Center. He received his Ph.D. in Geochemistry and Mineralogy from the Pennsylvania State University in 1984. He joined ORNL as a Eugene P. Wigner Fellow in 1983 and has spent his entire career there. His research interests center around the structures, dynamics, thermodynamics and kinetics of energy-relevant fluids and fluid-solid interfaces.

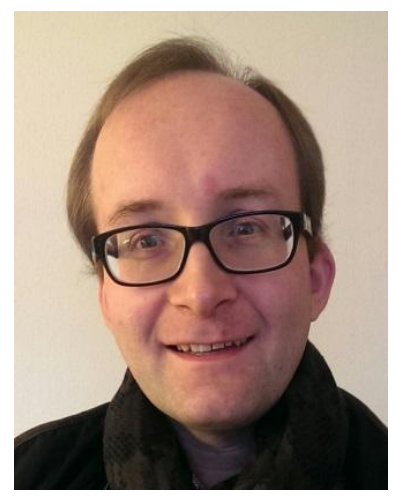

Sergei V. Kalinin is the director of the Institute for Functional Imaging of Materials (IFIM) and a distinguished research staff member at the Center for Nanophase Materials Sciences (CNMS) at Oak Ridge National Laboratory (ORNL). He received his PhD from the University of Pennsylvania in 2002, followed by Wigner fellowship at ORNL. He is the recipient of many international awards including the Presidential Early Career Award for Scientists and Engineers and the Burton medal of Microscopy Society of America. He studies the coupling between electromechanical, electrical and transport phenomena on the nanoscale and applications of big, deep, and smart imaging data in materials sciences. 


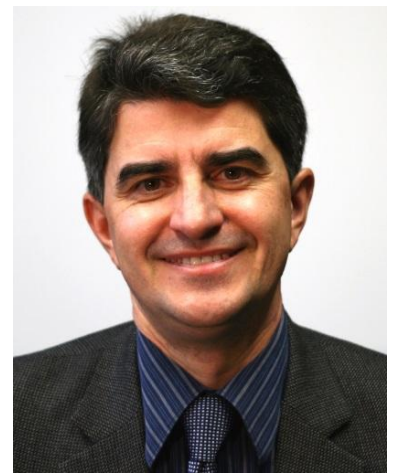

Yury Gogotsi is Distinguished University Professor and Trustee Chair of Materials Science and Engineering at Drexel University. He is also the founding Director of the A.J. Drexel Nanomaterials Institute and Associate Editor of ACS Nano. He has Ph.D. in Physical Chemistry from Kiev Polytechnic, D.Sc. in Materials Engineering from Ukrainian Academy of Sciences and Dr.h.c. degree from Toulouse University, France. He works on nanostructured carbons and two-dimensional carbides for energy related and biomedical applications. He was recognized as Highly Cited Researcher by Thomson-Reuters in 2014 and elected a Fellow of AAAS, MRS, ECS and ACerS and a member of the World Academy of Ceramics.

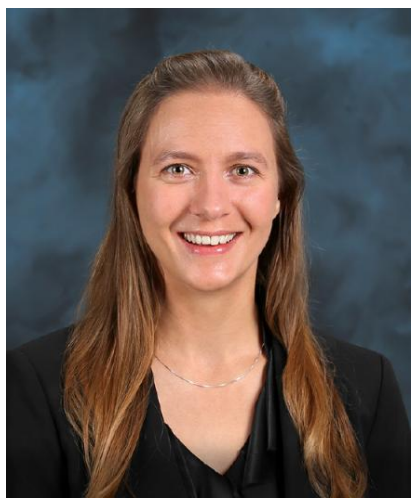

Nina Balke is a research staff member in the Center for Nanophase Materials Sciences (CNMS) at Oak Ridge National Laboratory. In 2006 she received her Ph.D in Materials Sciences from the Technical University of Darmstadt, Germany. After being a Feodor-Lynen fellow at the University of California in Berkeley she transitioned to CNMS in 2009 and is now working on nanoscale characterization of electromechanical effects in oxides focusing on energy storage materials. Her work was awarded the Department of Energy Early Career Research Award in 2011 and the American Ceramic Society's Robert L. Coble Award for Young Scholars in 2013. 


\section{Acknowledgements}

The experiments and sample preparation in this work were supported as part of the Fluid Interface Reactions, Structures and Transport (FIRST) Center, an Energy Frontier Research Center funded by the U.S. Department of Energy, Office of Science, Office of Basic Energy Sciences. This research was conducted at, and A. Rondinone supported for x-ray diffraction by, the Center for Nanophase Materials Sciences, which is a DOE Office of Science User Facility.

\section{References}

[1] P. Simon, Y. Gogotsi, Nat. Mater. 7 (2008) 845-854.

[2] F. Beguin, V. Presser, A. Balducci, E. Frackowiak, Adv. Mater. 26 (2014) 2219-2251, 2283.

[3] P. Simon, Y. Gogotsi, B. Dunn, Science. 343 (2014) 1210-1211.

[4] B. E. Conway, J. Electrochem. Soc. 138 (1991) 1539.

[5] V. Augustyn, J. Come, M. A. Lowe, J. W. Kim, P. L. Taberna, S. H. Tolbert, H. D. Abruna, P. Simon, B. Dunn, Nat. Mater. 12 (2013) 518-522.

[6] M. R. Lukatskaya, O. Mashtalir, C. E. Ren, Y. Dall'Agnese, P. Rozier, P. L. Taberna, M. Naguib, P. Simon, M. W. Barsoum, Y. Gogotsi, Science. 341 (2013) 1502-1505.

[7] M. Naguib, M. Kurtoglu, V. Presser, J. Lu, J. Niu, M. Heon, L. Hultman, Y. Gogotsi, M. W. Barsoum, Adv. Mater. 23 (2011) 4248-4253.

[8] M. Naguib, J. Come, B. Dyatkin, V. Presser, P. L. Taberna, P. Simon, M. W. Barsoum, Y. Gogotsi, Electrochem. Commun. 16 (2012) 61-64.

[9] M. Naguib, J. Halim, J. Lu, K. M. Cook, L. Hultman, Y. Gogotsi, M. W. Barsoum, J. Am. Chem. Soc. 135 (2013) 15966-15969.

[10] M. Ghidiu, M. Naguib, C. Shi, O. Mashtalir, L. M. Pan, B. Zhang, J. Yang, Y. Gogotsi, S. J. Billinge, M. W. Barsoum, Chem. Commun. 50 (2014) 9517-9520.

[11] M. Naguib, V. N. Mochalin, M. W. Barsoum, Y. Gogotsi, Adv. Mater. 26 (2014) 992-1005.

[12] J. Come, M. Naguib, P. Rozier, M. W. Barsoum, Y. Gogotsi, P. L. Taberna, M. Morcrette, P. Simon, J. Electrochem. Soc. 159 (2012) A1368-A1373.

[13] O. Mashtalir, M. Naguib, V. N. Mochalin, Y. Dall'Agnese, M. Heon, M. W. Barsoum, Y. Gogotsi, Nat. Commun. 4 (2013) 1716.

[14] M. D. Levi, M. R. Lukatskaya, S. Sigalov, M. Beidaghi, N. Shpigel, L. Daikhin, D. Aurbach, M. W. Barsoum, Y. Gogotsi, Adv. Energy Mater. 5 (2015) 1400815.

[15] R. H. Baughman, C. Cui, A. A. Zakhidov, Z. Iqbal, J. N. Barisci, G. M. Spinks, G. G. Wallace, A. Mazzoldi, D. De Rossi, A. G. Rinzler, O. Jaschinski, S. Roth, M. Kertesz, Science. 284 (1999) 13401344.

[16] G. Gu, M. Schmid, P. W. Chiu, A. Minett, J. Fraysse, G. T. Kim, S. Roth, M. Kozlov, E. Munoz, R. H. Baughman, Nat. Mater. 2 (2003) 316-319.

[17] M. Ghidiu, M. R. Lukatskaya, M. Q. Zhao, Y. Gogotsi, M. W. Barsoum, Nature. 516 (2014) 78-81.

[18] M. Kurtoglu, M. Naguib, Y. Gogotsi, M. W. Barsoum, MRS Communications. 2 (2012) 133-137.

[19] L. Y. Beaulieu, S. D. Beattie, T. D. Hatchard, J. R. Dahn, J. Electrochem. Soc. 150 (2003) A419A424.

[20] L. Y. Beaulieu, K. W. Eberman, R. L. Turner, L. J. Krause, J. R. Dahn, Electrochemical and Solid State Letters. 4 (2001) A137-A140.

[21] M. Inaba, T. Uno, A. Tasaka, J. Power Sources. 146 (2005) 473-477. 
[22] Z. Ogumi, M. Inaba, Bull. Chem. Soc. Jpn. 71 (1998) 521-534.

[23] G. H. Li, Y. Iijima, Y. Kudo, H. Azuma, Solid State Ionics. 146 (2002) 55-63.

[24] P. Haring, R. Kotz, G. Repphun, O. Haas, H. Siegenthaler, Appl. Phys. A: Mater. Sci. Process. 66 (1998) S481-S486.

[25] R. Nyffenegger, E. Ammann, H. Siegenthaler, R. Kotz, O. Haas, Electrochim. Acta. 40 (1995) 14111415.

[26] T. M. Arruda, M. Heon, V. Presser, P. C. Hillesheim, S. Dai, Y. Gogotsi, S. V. Kalinin, N. Balke, Energ. Environ. Sci. 6 (2013) 225-231.

[27] J. M. Black, G. Feng, P. F. Fulvio, P. C. Hillesheim, S. Dai, Y. Gogotsi, P. T. Cummings, S. V. Kalinin, N. Balke, Adv. Energy Mater. 4 (2014).

[28] A. Ganguly, Fundamentals of Inorganic Chemistry, second ed., Pearson Education, India, 2012.

[29] M. D. Levi, S. Sigalov, G. Salitra, R. Elazari, D. Aurbach, J. Phys. Chem. Lett. 2 (2011) 120-124.

[30] T. Ohkubo, T. Konishi, Y. Hattori, H. Kanoh, T. Fujikawa, K. Kaneko, J. Am. Chem. Soc. 124 (2002) 11860-11861.

[31] M. Winter, G. H. Wrodnigg, J. O. Besenhard, W. Biberacher, P. Novak, J. Electrochem. Soc. 147 (2000) 2427-2431.

[32] M. M. Hantel, V. Presser, R. Kötz, Y. Gogotsi, Electrochem. Commun. 13 (2011) 1221-1224.

[33] M. M. Hantel, V. Presser, J. K. McDonough, G. Feng, P. T. Cummings, Y. Gogotsi, R. Kotz, J. Electrochem. Soc. 159 (2012) A1897-A1903.

[34] M. M. Hantel, T. Kaspar, R. Nesper, a. Wokaun, R. Kötz, Electrochem. Commun. 13 (2011) 90-92.

[35] J. Swiatowska-Mrowlecka, V. Maurice, L. Klein, P. Marcus, Electrochem. Commun. 9 (2007) 24482455.

[36] D. Aurbach, B. Markovsky, A. Rodkin, E. Levi, Y. S. Cohen, H. J. Kim, M. Schmidt, Electrochim. Acta. 47 (2002) 4291-4306.

[37] L. Y. Beaulieu, T. D. Hatchard, A. Bonakdarpour, M. D. Fleischauer, J. R. Dahn, J. Electrochem. Soc. 150 (2003) A1457-A1464.

[38] M.-Q. Zhao, C. E. Ren, Z. Ling, M. R. Lukatskaya, C. Zhang, K. L. Van Aken, M. W. Barsoum, Y. Gogotsi, Adv. Mater. (2014) 1-7.

[39] Z. Ling, C. E. Ren, M.-Q. Zhao, J. Yang, J. M. Giammarco, J. Qiu, M. W. Barsoum, Y. Gogotsi, Proc. Natl. Acad. Sci. U. S. A. 111 (2014) 16676-16681.

[40] J. Torop, V. Palmre, M. Arulepp, T. Sugino, K. Asaka, A. Aabloo, Carbon. 49 (2011) 3113-3119. 
a)
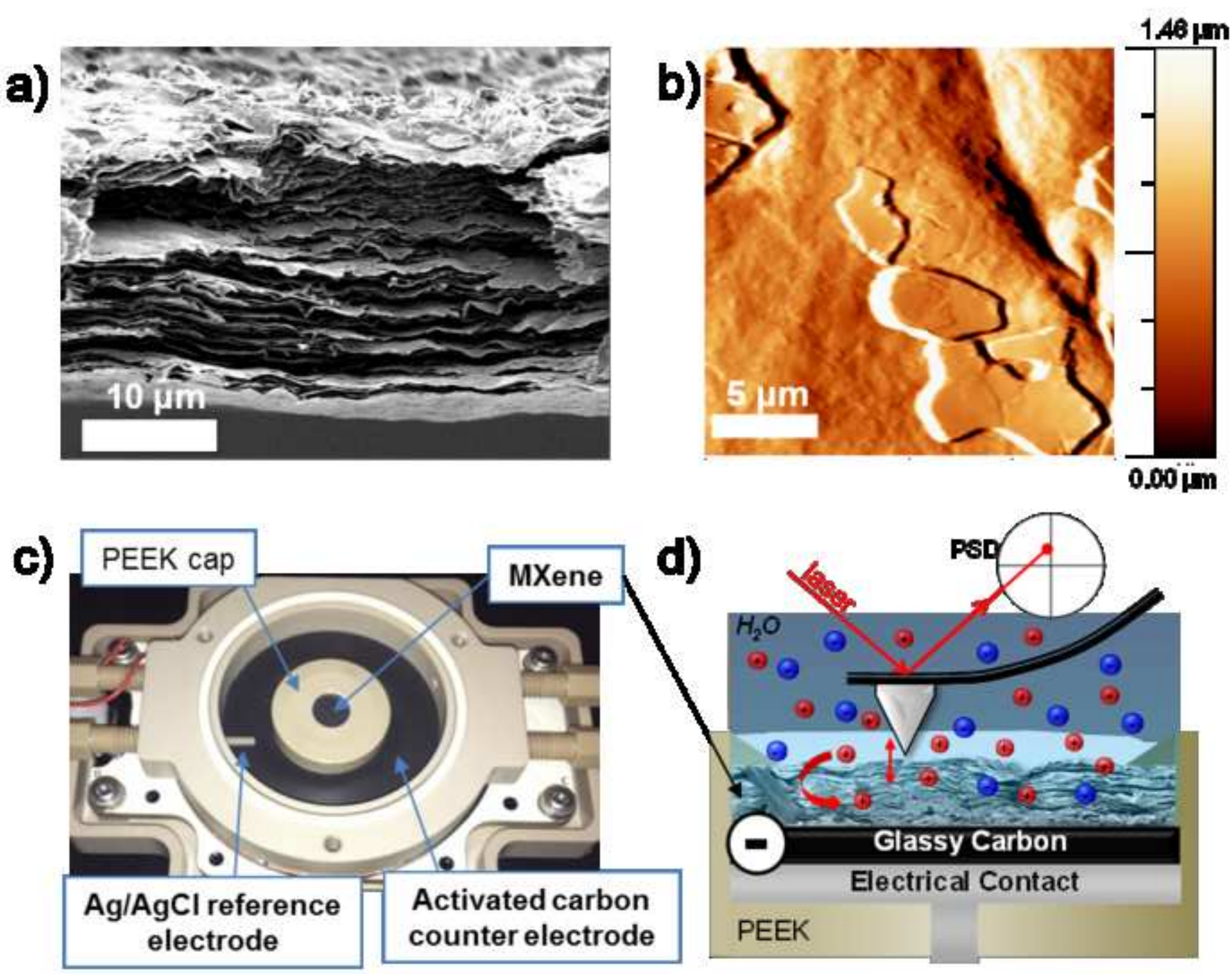

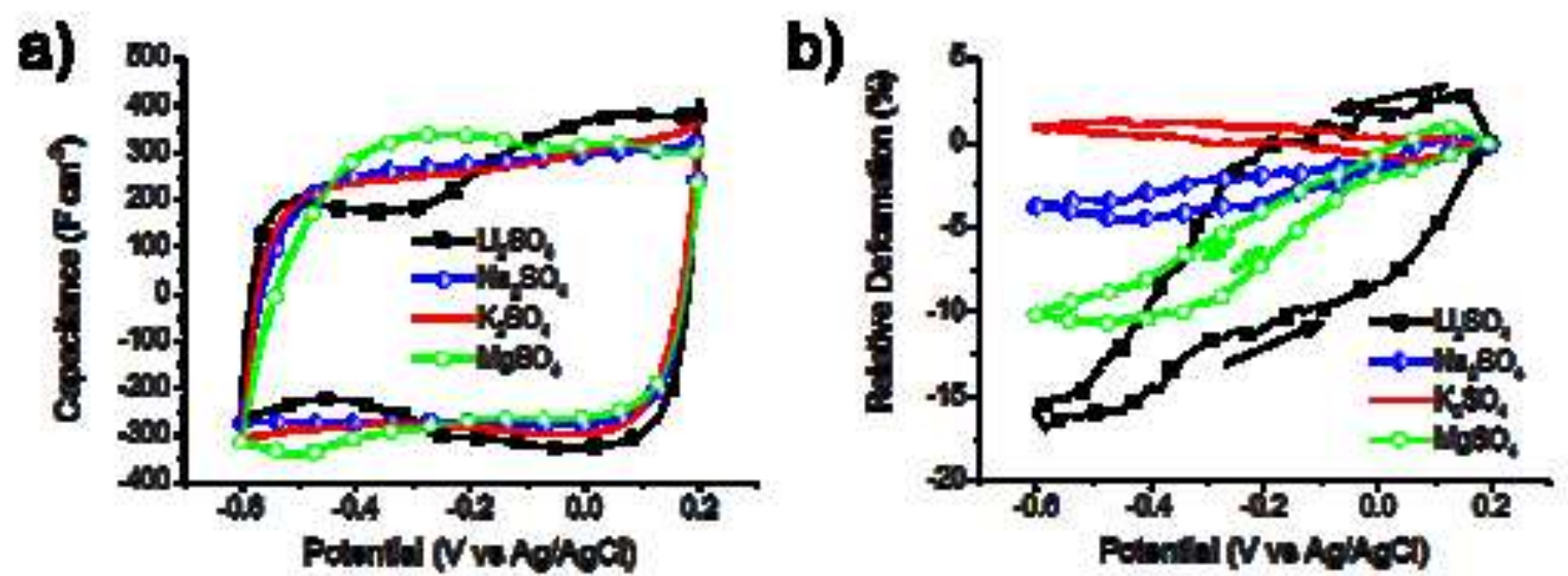

c)

Wegatholy eh ged anthes

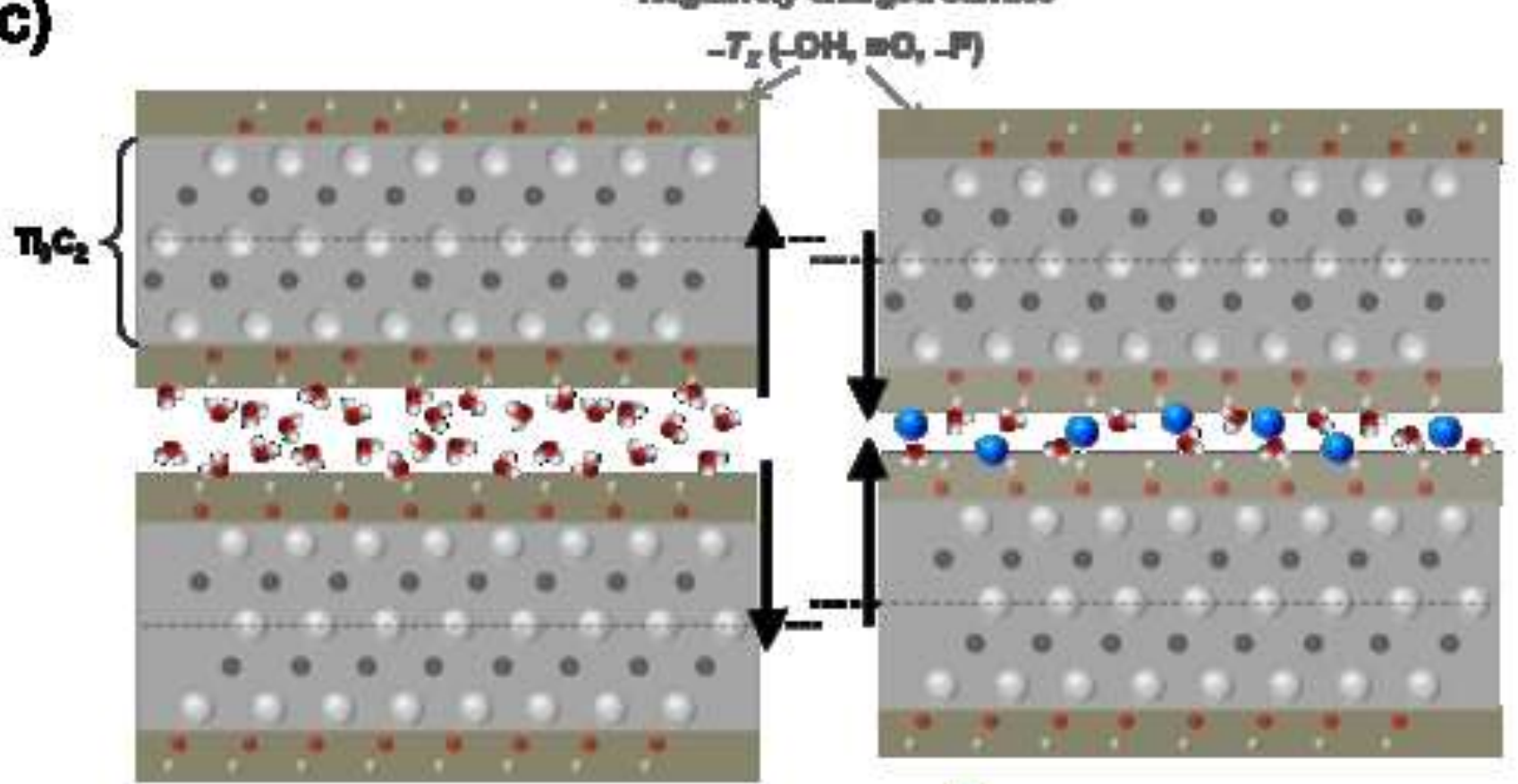

Q 1 O Oation

Charged stato

- C $\mathrm{H}$ 
Figure 3

a)

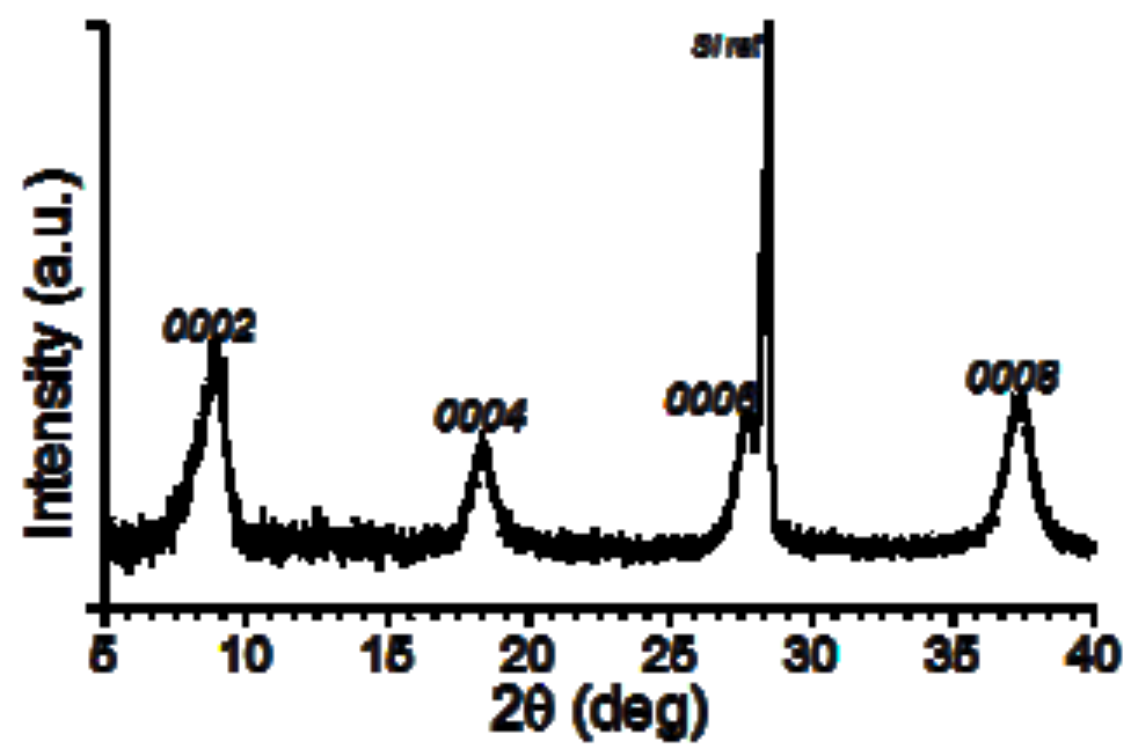

b)

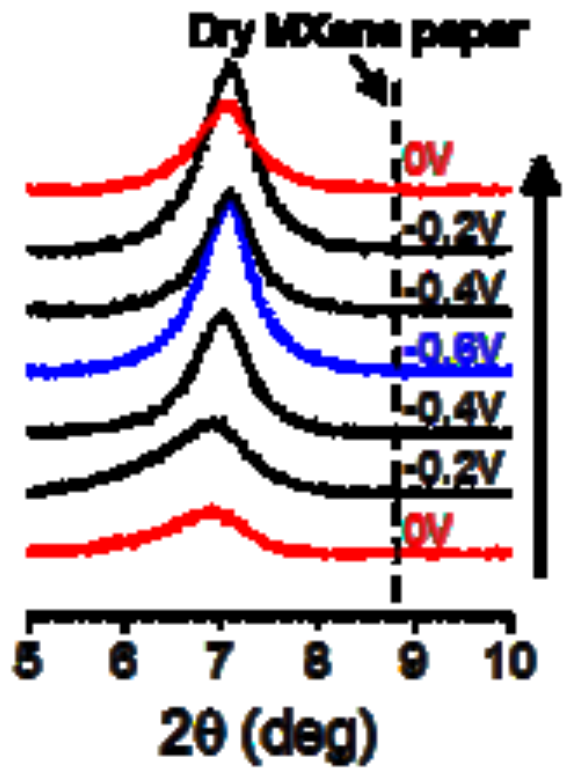

c)
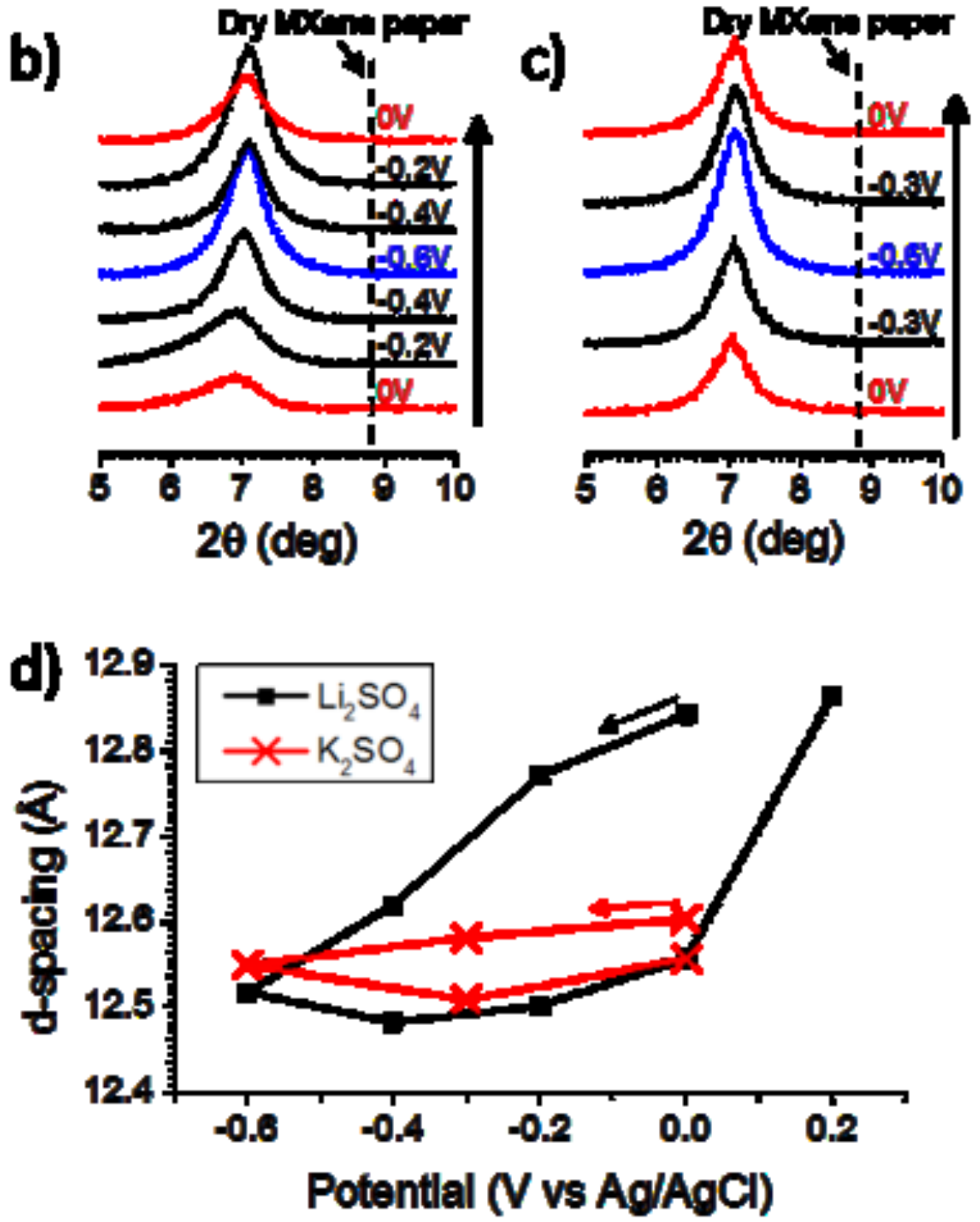

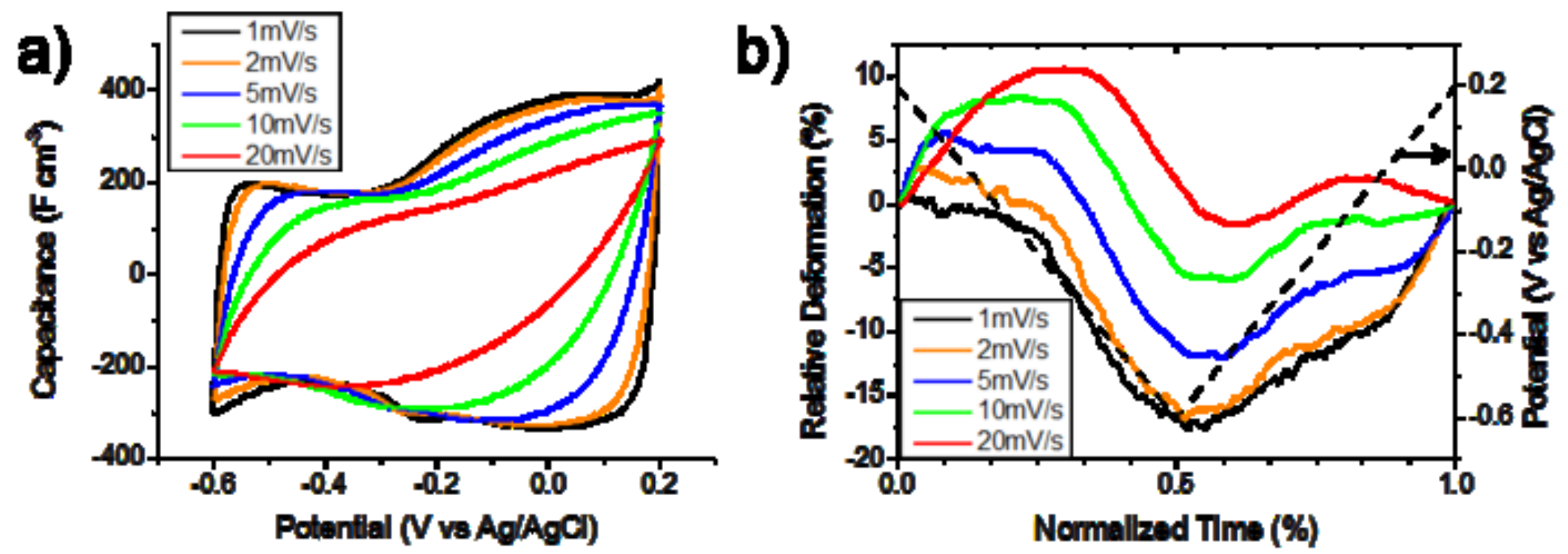
Figure 5
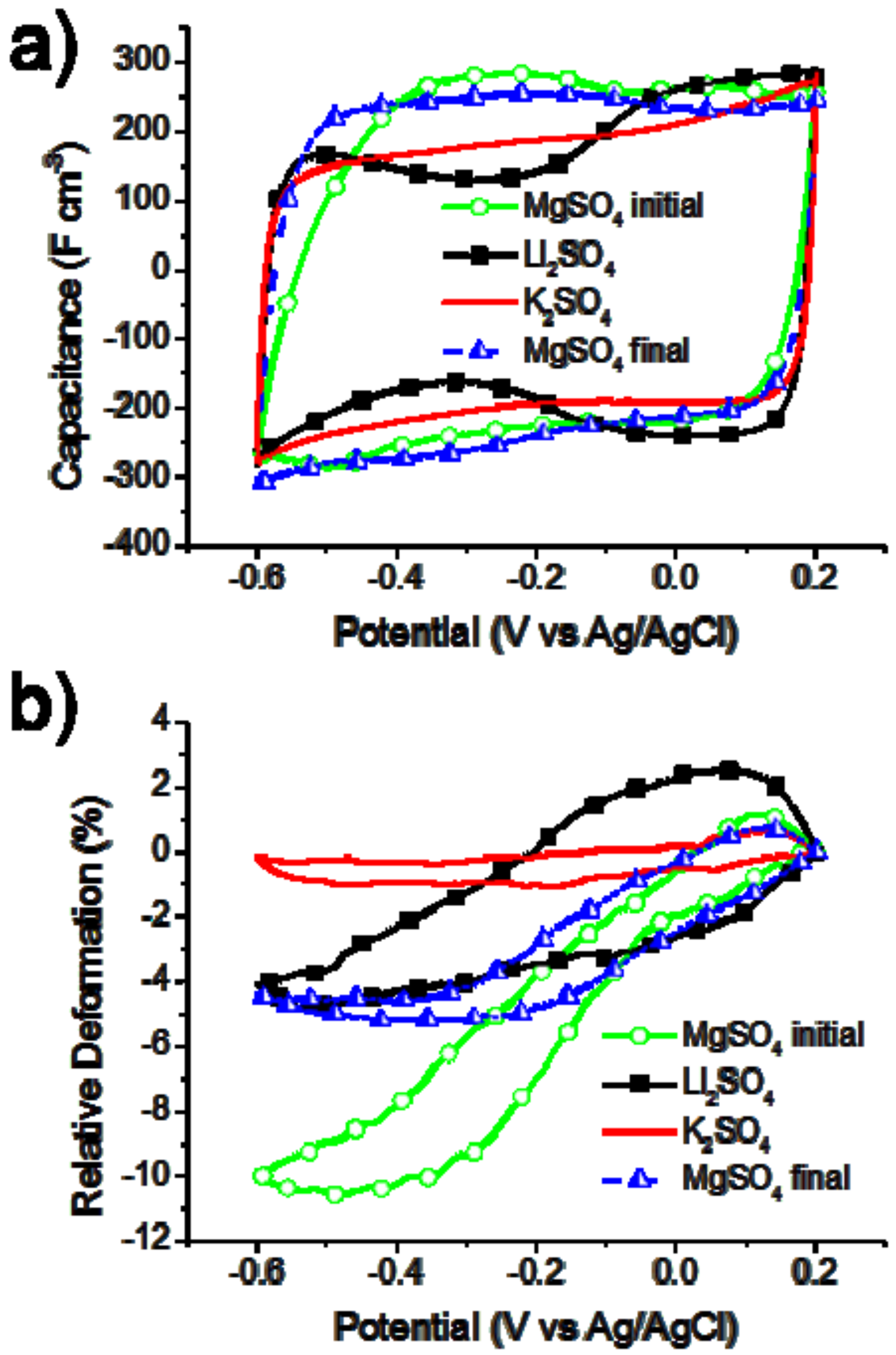


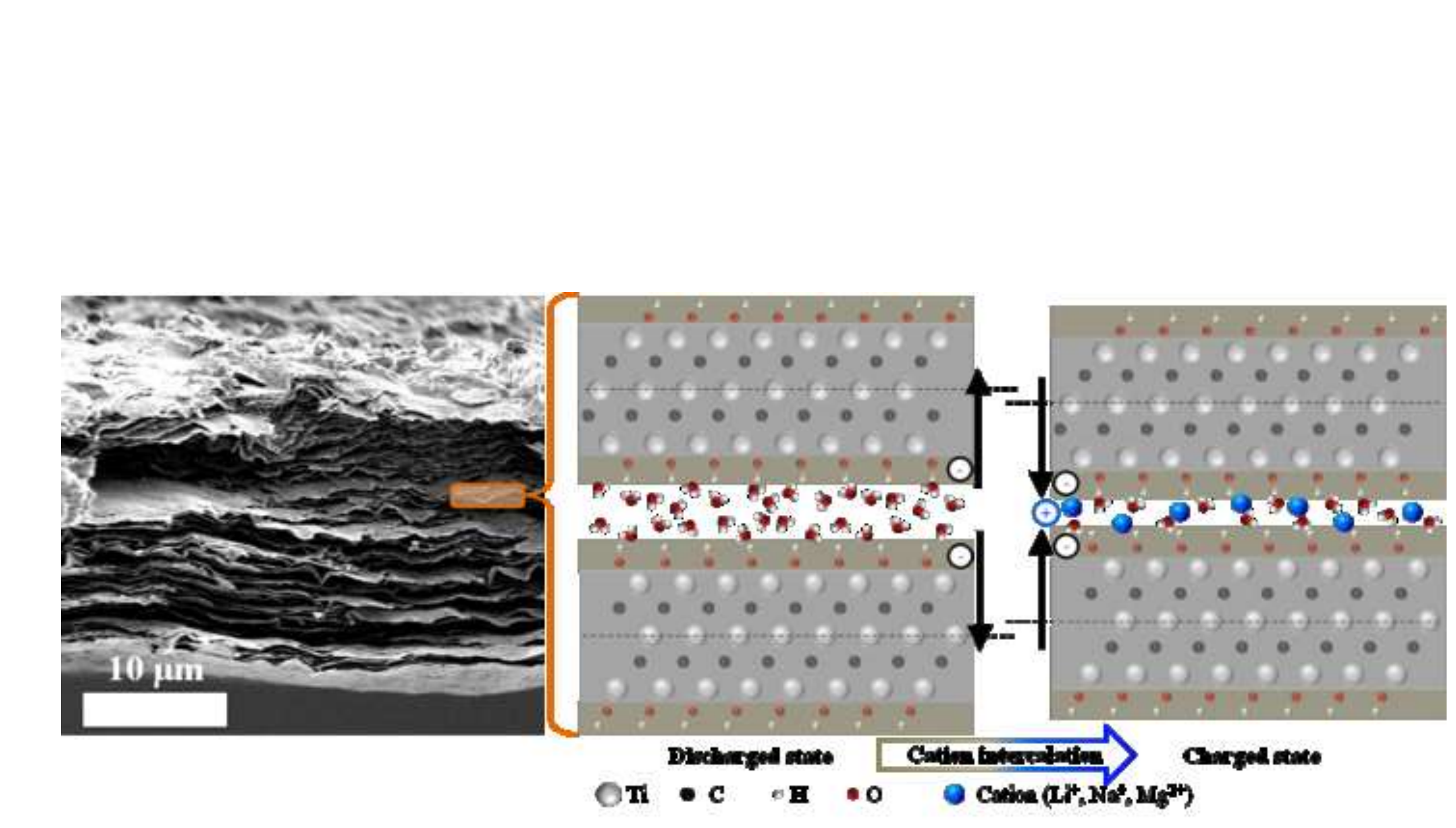

